\title{
ESTANDARIZACIÓN DE PROCESOS ADMINISTRATIVOS DEL ÁREA DE GESTIÓN HUMANA, SEGURIDAD Y SALUD EN EL TRABAJO EN UNA ENTIDAD ONCOLÓGICA ${ }^{1}$
}

\section{Standardization of administrative processes in the area of human management, safety and health at work in an oncological entity}

EVER ÁNGEL FUENTES ROJAS MBA ${ }^{2}$, FABIO ANDRÉS CORDERO USECHE ${ }^{3}$, INGRID DANIELA GÓMEZ ARÉVALO 4

Recibido:29 de mayo de 2020. A ceptado:15 de mayo de 2020

D OI: http://dx.doi.org/10.21017/rimci.2020.v7.n14.a85

\begin{abstract}
Resumen
En el mercado organizacional actual, para que una empresa progrese de manera exponencial, el área de G estión Humana deberá ejercer un papel primordial, dentro de sus funciones principales está procurar al máximo la satisfacción y el crecimiento de los colaboradores en sus áreas de conocimiento y hacerles saber que cada uno de ellos es importante y necesario para obtener excelentes resultados; se requiere de una buena gestión que esté bien estructurada para permitir el hallazgo de posibles fallas internas, estas circunstancias dan lugar a la búsqueda de estrategias que posibiliten la mejora de los procedimientos ejecutados en la entidad oncológica, por lo anterior se da lugar a la necesidad de incorporar la estandarización del área, con relación a esto se plantearon los siguientes objetivos: diagnosticar la situación actual de la gestión documental de los procesos involucrados en el área de G estión Humana y Sistema de Gestión de Seguridad y Salud en el Trabajo, desarrollar un estudio de tiempos y movimientos de las operaciones para el mejoramiento en la prestación del servicio, documentar los procesos que se llevan a cabo en el área, realizar un ajuste y complemento de indicadores para que permitan medir los resultados obtenidos y por último realizar un análisis de impacto para la determinación de la viabilidad del proyecto.

Para esta investigación, se aplicaron herramientas de ingeniería tales como el diseño y aplicación de entrevistas no estructuradas, matriz de Vester para priorizar los problemas, uso de un listado maestro para visualizar todos los documentos presentes en el área, uso de tiempos estructurados, ajuste de indicadores de gestión y matriz de Leopold para medir el impacto del proyecto; dentro de los beneficios generados, se resalta la disminución de la duplicidad de actividades, la reducción de errores y el aporte a la adherencia de los colaboradores a la entidad.
\end{abstract}

Palabras clave. Estandarización; desarrollo; beneficios; metas; indicadores; impacto.

\begin{abstract}
In the current organizational market, for a company to progress exponentially, the Human Management area must play a primary role, within its main functions is to ensure maximum satisfaction and growth of employees in their areas of knowledge and make them know that each of them is important and necessary to obtain excellent results; Good management is required that is well structured to allow the discovery of possible internal failures, these circumstances give rise to the search for strategies that make it possible to improve the procedures performed in the oncological entity, therefore, the need arises to incorporate the standardization of the area, in relation to this the following objectives were
\end{abstract}

1 A rtículo basado en el proyecto de estandarización de procesos desarrollado en una entidad que se enfoca en la promoción, prevención y detección temprana del cancer para el área de Talento Humano y Seguridad y Salud en el Trabajo, ejecutado en los años 2018,2019 y 2020 por estudiantes del programa de ingeniería industrial de la Universidad Libre, sede Bogotá

2 Ingeniero industrial. Universidad Libre, Colombia. Docente de jornada completa del programa de ingeniería industrial. U niversidad Libre, Colombia. M agister in Business A dministrator. ORCID : https://orcid.org/0000-0001-9671-5884 Correo Electrónico: ever.fuentes@ unilibre.edu.co

3 Estudiante de ingeniería industrial. U niversidad Libre, Colombia

4 Estudiante de ingeniería industrial. Universidad Libre, Colombia

Rev. Ingeniería, M atemáticas y Ciencias de la Información

Vol. 7 / Núm. 14 / julio - diciembre de 2020; pág. 77-93 
raised: diagnose the current situation of document management of the processes involved in the area of Human Management and the 0 ccupational Health and Safety Management System, develop a study of times and movements of operations for the improvement in the provision of the service, document the processes that are carried out in the area, make an adjustment and complement of indicators so that they allow to measure the results obtained and finally carry out an impact analysis to determine the fea sibility of the project. For this research, engineering to ols were applied such as the design and application of unstructured interviews, Vester matrix to prioritize problems, use of a master list to view all documents present in the area, use of structured times, adjustment of management indicators and Leopold matrix to measure the impact of the project; Among the benefits generated, the reduction in duplication of activities, the reduction of errors and the contribution to the adherence of the collaborators to the entity are highlighted.

Key words. Standardization; developing; benefits; metas; indicators; impact.

\section{INTRODUCCIÓN}

ARA SER una empresa competitiva, además de tener orden y limpieza externa, se debe conseguir el "orden interno", este orden no es otra cosa que la estandarización de procesos, trabajar de manera estandarizada consiste en establecer una manera organizada y controlada de laborar [1], al tener las actividades normalizadas, no solo se contribuye al cumplimiento de requisitos legales, también se facilita la revisión de auditoría, puesto que se posee una integridad en los documentos, todo lo anterior para mantener la coherencia estipulada en el desarrollo estratégico de la organización.

La asociación de derecho privado sin ánimo de lucro elegida para realizar el estudio tiene como misión la prevención y transformación dela experiencia del cáncer en Colombia, se comprometen con el paciente, su familia y la comunidad, cabe resaltar que asimismo se realizan acciones de educación, prevención y diagnóstico temprano del cáncer con participación de voluntariado. Aunque el índice desupervivencia frentea una detección anticipada es bastante alto, muchos países no cuentan con los recursos suficientes para responder esta necesidad, la organización en mención busca promover estilos de vida saludables y una vez detectada la patología, se ofrecen servicios de quimioterapia, consulta externa, farmacia y asesorías terapéuticas a pacientes y familiares para afrontar de una forma más asertiva la situación.

Debido a los cambios organizacionales presentados en los últimos años, la entidad manifestó la necesidad de desarrollar ajustes y actualizaciones en los procesos, contemplando la estandarización como una medida necesaria, es por esto que la finalidad principal serála normalización del área de Gestión H umana y Seguridad y Salud en el Trabajo con el fin de dar cumplimiento a los objetivos estipulados en cada procedimiento.
El artículo mencionalos pasos por los quepasó la estandarización, siendo el primero el diagnóstico inicial de la documentación, luego el ajuste, creación o actualización delos documentos del área, posteriormenteel estudio detiempos y movimientos deoperaciones, el ajuste de los indicadores que miden y controlan las tareas y, por último, el estudio de impacto que determina la viabilidad del proyecto.

Como primer paso serealizó el diagnóstico, por medio del diseño y validación de entrevistas no estructuradas a la subdirectora del área, con el fin de obtener una visión general de la gestión documental, es decir, determinar cuántos procedimientos, manuales, formatos y anexos existían en ese momento y cuántos hacían falta por actualizar; para complementar esta fase, se realizó la caracterización, donde se define el objetivo, alcance, proveedores, entradas, salidas y relación con los clientes, con base en esta información, se elaboró el mapa deriesgos, identificando el tipo de riesgo, las causas, los efectos, la severidad y los controles para luego validar de forma gráfica los riesgos residuales e inherentes.

En la fase de la documentación, como referente seutilizó un listado maestro, el cual menciona todas las actividades con sus respectivos códigos y versiones, estedocumento facilitó la actualización y el ajuste de los procedimientos originales y la creación de Ios faltantes, los cuales tienen objetivo, pasos deejecución, un diagrama deflujo quedescribedemanera gráfica el orden de cumplimiento y sus respectivos anexos, manuales o formatos relacionados.

Para la tercera fase que consistió en el estudio de tiempos y movimientos, se usó la metodología de tiempos estructurados, esta actividad consiste en real izar una encuesta a la subdirectora del área y a dos expertos que laboren en sectores relacionados con la organización, quienes brindan una duración estimada a cada tarea de cada procedi- 
miento, los resultados brindan un “tiempo estándar" que es analizado y permite real izar una comparación para establecer la productividad de cada proceso calificado.

Con baseen la información anterior, seestablece el ajuste y complemento de los indicadores de gestión que tienen como función medir el cumplimiento de los objetivos y metas del área de Gestión Humana y Seguridad y Salud en el Trabajo; finalmente, para el estudio de impacto, se realizó la matriz de Leopold que refleja los beneficios positivos y negativos del caso de estudio.

\section{M ARCO TEÓRICO}

A continuación, se muestran los conceptos y teorías que seutilizaron como guía para el cumplimiento de los objetivos:

\section{A. Estandarización}

“La estandarización de hoy.... Es el fundamento necesario en el que se basa la mejora de mañana. Si uno piensa en la-estandarización-como aquello que refleja la mejor práctica que se conoce hoy en día, pero quese mejorará mañana...Ilegará lejos. Pero si uno piensa en los estándares como algo limitador, entonces se parará el progreso." (Henry Ford). Se gún "Tiziana Ingrande" la estandarización de tareas y procesos es uno de los fundamentos de la mejora continua", esto mediante la reducción de la variabilidad en un proceso, a través dela documentación y capacitación del personal acerca de la me jor forma para desarrollar sus actividades [2].

El proceso deestandarización según el "Productivity Press Development Team (2002)" está definido en cuatro pasos presentados a continuación:

- Definir el estándar

- Informar el estándar

- Establecer la adhesión al estándar

- Proporcionar una mejora continua del estándar

La estandarización es un proceso que requiere esfuerzo y su dificultad está implicada en que, al ser un cambio dentro de la organización, requiere de tiempo y compromiso para su correcto desempeño, pero tiene grandes contribuciones en la empresa como lo son, el aumento de la productividad, la poca variabilidad desus procesos, Ia continuidad a través del tiempo y la reducción de pérdidas [3].

\section{B. Levantamiento de procesos}

Cada uno de los procesos se conforma de cinco partes claves que deben ser tratadas a cabalidad para conseguir su correcta documentación, los cuales son:

- El objetivo del proceso

- Las actividades

- El producto del proceso o resultado final

- Los indicadores de gestión

- Los controles del proceso

La unión sólida mediantela documentación de las anteriores partes genera en el proceso eficacia y la mejora continua. Es importante entender que para el levantamiento de procesos se deben tener en cuenta los siguientes pasos:

- Entendimiento de los Procesos/ Subprocesos de Negocio: En esta etapa se debe proceder a realizar una descripción de los procesos. Cada proceso se encuentra conformado por una serie de procedimientos, y estos a su vez por actividades o tareas por desarrollar. Para realizar una adecuada descripción de los procesos, procedimientos y actividades institucionales debe contarse con un conocimiento preciso y claro de los mismos, por ello es importante utilizar técnicas de levantamiento de información con los colaboradores claves. Se utilizarán técnicas de observación, entrevista y focus group para todo el levantamiento deinformación

- Identificación de etapas y actividades específicas delos procesos y subprocesos: En esta etapa se identifica cada etapa, subproceso o procedimiento que debe ser registrado

- Generar fichas y diagramas de flujo de los procesos: En esta etapa se describirán detaIladamente cada proceso, subproceso y/ o procedimiento y sedocumentarála forma en que se llevan a cabo a través de fichas y diagramas deflujo connotación BPMN 
- Establecer indicadores de gestión: Estos indicadores y KPIs serán usados para el control de gestión y retroalimentación de la mejora continua de los procesos [4].

\section{Capital Humano}

Es el aumento en la capacidad dela producción del trabajo al canzada con mejoras en las capacidades de trabajadores. Estas capacidades realzadas se adquieren con el entrenamiento, la educación y la experiencia. Serefiereal conocimiento práctico, las habilidades adquiridas y las capacidades aprendidas deun individuo quelo hacen potencial mente. En sentido figurado se refiereal término capital en su conexión con lo que quizá sería mejor Ilamada la "calidad del trabajo" [5].

\section{D . I mportancia del desarrollo del capital humano en la empresa}

Se sabe que la tecnología de avanzada es indispensable para lograr la productividad quehoy exige el mercado, pero se observa que el éxito de cualquier emprendimiento se depende principalmente a la flexibilidad y de la capacidad de innovación que tenga la gente que participa en la organización.

A demás, en la era actual, la tecnología y la información están al alcance de todas las empresas, por lo quela única ventaja competitiva que puedediferenciar una organización deotra es la capacidad que tienen las personas dentro de la organización de adaptarse al cambio. Esto se logra mediante el fortalecimiento dela capacitación y aprendizaje continuo en las personas a fin de que la educación y experiencias sean medibles y más aún, valorizadas conforme a un sistema de competencias [6].

\section{E. Indicador}

Son variables que intentan medir u objetivar en forma cuantitativa o cual itativa, sucesos colectivos para así, poder respaldar acciones [7].

\section{F. Proceso}

Sucesión einterrelación depasos, tareasy decisiones con valor agregado, que se vinculan entre sí para transformar un insumo en un producto o servicio [8].

\section{G . Bienestar laboral}

Forma parte de la administración del Talento Humano y hace parte del conjunto de programas y beneficios que seestructuran como solución a las necesidades del individuo, influye como el emento importante dentro de una comunidad funcional o empresa a la que se pertenece.

\section{H . Riesgo}

Efecto de la incertidumbre, que es el estado de la deficiencia de información, relacionado con la compresión o conocimiento de un evento, consecuencia y probabilidades [9].

\section{M apa de riesgos}

Es una herramienta basada en los distintos sistemas de información que pretende identificar las actividades o procesos sujetos a riesgo. Además, este mapa de riesgos cuantifica la probabilidad de que estos eventos sucedan y mide el daño potencial en caso de que dicho riesgo suceda [10].

\section{J. Estudio de tiempos}

Es el proceso donde se mide el tiempo requerido para terminar una actividad a una velocidad normal [11].

\section{K. Tiempo estándar}

Tiempo requerido para que un operario de tipo medio, plenamente cal ificado y adiestrado, y trabajando a un ritmo normal, Ilevea cabo su trabajo [11].

\section{D iagramas de operaciones}

Brinda una idea del flujo de trabajo del proceso, lo que ayudará a comprender el proceso y su funcionamiento e identificar secuencias de eventos, puntos de decisión y tiempos de espera [12].

\section{M.G estión del talento humano}

Es la estrategia de una organización para la contra tación, capacitación y retención delos empleados [13].

\section{N . G estión}

Actividades coordinadas para dirigir y controlar una organización [14].

Rev. Ingeniería, M atemáticas y Ciencias de Ia Información Vol. 7 / N úm. 14 / julio - diciembre de 2020; pág. 77-93 


\section{Sistema de gestión de seguridad y salud en el trabajo}

Se basa en la mejora continua, lo cual incluye la política, la organización, la planificación, la aplicación, la evaluación, la auditoría y las acciones de mejora con el objetivo de anticipar, reconocer, evaluar y controlar los riesgos que puedan afectar la seguridad y la salud en los espacios laborales [15].

\section{P. O portunidades de mejora}

Son aquellas que permiten afianzar el crecimiento y competitividad a través dela planeación y ejecución de actividades que permitan mitigar inconvenientes descubiertos en acciones de inspección [16].

\section{Q. A uditoría}

Se definecomo un proceso sistemático de obtener y evaluar los registros con el fin deverificar su estado y proponer oportunidades de mejora [17].

\section{R. Requisitos legales}

Es el conjunto de leyes, decretos, resoluciones, normas, estatutos, etc, que sean aplicables al proceso llevadas a cabo por cada una de las dependencias del área [18].

\section{S. Nómina}

Es el registro financiero que una empresa real iza sobre los salarios de sus empleados, bonificaciones y deducciones [19].

\section{Antecedentes}

En la tabla I, se visualizan las referencias de casos de estudio similares que se utilizaron en el desarrollo de este proyecto.

\section{Metodología}

Con base en el estudio de los antecedentes relacionados con metodologías de implementación dela estandarización en organizaciones similares al caso de estudio, se identifica que la investiga- ción aplicada en el desarrollo del proyecto es de tipo descriptivo, pues la actividad principal del proyecto es especificar y detallar de manera cuantitativa o cualitativa, a partir de entrevistas y toma de tiempos los procesos presentados en el área de Gestión Humana y Seguridad y Salud en el Trabajo.

Una investigación de tipo descriptiva se utiliza para detallar de forma sistemática y precisa los hechos y características de una población o área de interés determinada, especificar las características delas personas, situaciones o grupos y la frecuencia con la que ocurren ciertos fenómenos, también para descubrir asociaciones o relaciones entrevariables seleccionadas y por último responder a preguntas basadas en los eventos en curso del presente [30].

En la figura 1 se visualizan los pasos y actividades que se tomaron en cuenta para el desarrollo del caso de estudio

\section{Resultados}

Como se mencionaba al inicio de este artículo, los procesos de Gestión Humana y Seguridad y Salud en el Trabajo semanejaban de manera independiente. Al ejecutar el proyecto, estas áreas se unificaron y decidieron adicionar el área de Ambiente, sin embargo, después de acuerdos organizacionales y estratégicos internos, se comunicó la decisión de nombrar el proceso como Gestión Humana y transferir el proceso de A mbiente a otra área, lo cual suprimió varios documentos del alcance.

Para la ejecución del proyecto, los mecanismos empleados fueron los siguientes:

\section{A. D iagnóstico del área}

Primero, se realizó una entrevista no estructurada a la persona encargada del área de Gestión Humana y Seguridad y Salud en el Trabajo; este tipo de encuentro, también Ilamado entrevista de profundidad consiste en hacer preguntas para recopilar datos sobre un tema, tiene menor número de interrogantes ya que se inclina más hacia una conversación normal [31]. 
Tabla I. Antecedentes.

\begin{tabular}{|c|c|c|}
\hline Autores & Título & Descripción \\
\hline $\begin{array}{l}\text { Tania Verónica } \\
\text { Guachi } \\
\text { Aucapiña, David } \\
\text { Omar Guevara } \\
\text { Aulestia }\end{array}$ & $\begin{array}{l}\text { Norma de seguridad } \\
\text { informática ISO } 27001 \\
\text { para mejorar la } \\
\text { confidencialidad, } \\
\text { integridad y } \\
\text { disponibilidad de los } \\
\text { sistemas de } \\
\text { información y } \\
\text { comunicación en el } \\
\text { departamento de } \\
\text { sistemas de la } \\
\text { Cooperativa de Ahorro } \\
\text { y Crédito San Francisco } \\
\text { LTDA }\end{array}$ & $\begin{array}{l}\text { Con el fin de obtener un diagnóstico completa sobre el estado del área de } \\
\text { sistemas de la Cooperativa de Ahorro y Crédito San Francisco LTDA., el } \\
\text { enfoque de la investigación fue cualitativo, ya que esta se basó en la } \\
\text { información obtenida por medio de entrevistas y por consiguiente se } \\
\text { realizaron análisis y se logró proponer diferentes alternativas de } \\
\text { solución; de esta manera se establecieron tres tipos de investigación: } \\
\text { exploratorio, descriptivo y de asociación de variables; el primero se usó } \\
\text { para determinar las condiciones actuales de la seguridad de los sistemas } \\
\text { de información y de comunicación, seguido del descriptivo que fue útil } \\
\text { para conocer con profundidad el problema, estableciendo sus causas y } \\
\text { consecuencias así como las dificultades por lo que está atravesando y por } \\
\text { último se determinó la relación de una variable con la otra y la incidencia } \\
\text { que tiene en la solución del problema [20]. }\end{array}$ \\
\hline $\begin{array}{c}\text { Martínez } \\
\text { Guerrero José } \\
\text { Miguel, Silva } \\
\text { Delgado Camilo } \\
\text { Andrés }\end{array}$ & $\begin{array}{c}\text { Guía metodológica para } \\
\text { el levantamiento y } \\
\text { análisis de } \\
\text { requerimientos de } \\
\text { software con base en } \\
\text { procesos de negocio }\end{array}$ & $\begin{array}{l}\text { La metodología que se usó en este proyecto fue la investigativa divida en } \\
\text { cinco fases, cada una con sus propias características y metodología las } \\
\text { cuales se plantearon inicialmente de la siguiente manera: } \\
\text { Fase I: Investigación de los procesos } \\
\text { Fase II: Revisión de la fase anterior para determinar los componentes de } \\
\text { las arquitecturas } \\
\text { Fase III: Análisis } \\
\text { Fase IV: Se crea metodología enfocada a los procesos } \\
\text { Fase V: Se procede a implementar la guía metodológica [21] }\end{array}$ \\
\hline $\begin{array}{l}\text { Alberto, Julián } \\
\text { Andrés, } \\
\text { Monsalve-pulido } \\
\text { Fredy } \\
\text { Fernando, } \\
\text { Aponte- Novoa } \\
\text { David }\end{array}$ & $\begin{array}{l}\text { Estudio y gestión de } \\
\text { vulnerabilidades } \\
\text { informáticas para una } \\
\text { empresa privada en el } \\
\text { departamento de } \\
\text { Boyacá }\end{array}$ & $\begin{array}{l}\text { La seguridad de la información digital para una organización depende } \\
\text { de diferentes frentes: el físico, referente al alojamiento de la información; } \\
\text { el social, relacionado con el grado de discrecionalidad del personal que } \\
\text { la manipula, y el lógico, que se refiere a la configuración de sus niveles } \\
\text { de accesibilidad y disposición. Es así como técnicamente se ha } \\
\text { caracterizado que un esquema seguro debe corresponder al ajuste de los } \\
\text { niveles de confidencialidad, integridad y disponibilidad de la } \\
\text { información, según la norma ISO } 27001 \text {. Cuando se describen las } \\
\text { anteriores características, se encuentran implícitas en la información, por } \\
\text { lo que las organizaciones deben complementar el ciclo de seguridad con } \\
\text { la constitución de marcos efectivos para que su información fluya dentro } \\
\text { de procesos bien estructurados, constituyendo los denominados } \\
\text { Sistemas de Gestión de Seguridad de la Información (SGSI) [22]. }\end{array}$ \\
\hline $\begin{array}{l}\text { Aura Lucia } \\
\text { Casadiegos } \\
\text { Santana } \\
\text { Marcela } \\
\text { Quintero } \\
\text { Jiménez } \\
\text { Mileidy Toro } \\
\text { Rueda }\end{array}$ & $\begin{array}{l}\text { Sistema de gestión de } \\
\text { seguridad de la } \\
\text { información (SGSI) } \\
\text { para el área de } \\
\text { contabilidad de la E.S.E. } \\
\text { Hospital Local de Rio } \\
\text { de Oro Cesar }\end{array}$ & $\begin{array}{l}\text { COBIT es un framework (También llamado marco de trabajo) de } \\
\text { Gobierno de TI y un conjunto de herramientas de soporte para el } \\
\text { gobierno de TI que les permite a los gerentes cubrir la brecha entre los } \\
\text { requerimientos de control, los aspectos técnicos y riesgos de negocio. } \\
\text { Describe como los procesos de TI entregan la información que el negocio } \\
\text { necesita para lograr sus objetivos, los tres componentes claves son: los } \\
\text { recursos de TI, los criterios de información y los procesos de TI [23]. }\end{array}$ \\
\hline $\begin{array}{l}\text { Ricardo Gómez, } \\
\text { Diego Hernán } \\
\text { Pérez, Yesid } \\
\text { Donoso, Andrea } \\
\text { Herrera }\end{array}$ & $\begin{array}{c}\text { Metodología y gobierno } \\
\text { de la gestión de riesgos } \\
\text { de tecnologías de la } \\
\text { información }\end{array}$ & $\begin{array}{l}\text { El riesgo ha existido inherente a cada acción que realiza el ser humano. } \\
\text { Sin embargo, en la sociedad actual, inmersa en un ambiente altamente } \\
\text { tecnológico y donde la información es el centro de las actividades, se ha } \\
\text { desarrollado una creciente dependencia de las TI lo que las ha } \\
\text { convertido en un gran factor de riesgo y quizás, uno de los más } \\
\text { importantes de este siglo; Se hace necesario, entonces, contar con } \\
\text { metodologías que faciliten el logro de estos objetivos de altos volúmenes } \\
\text { de información. OCTAVE (Operationally Critical Threat, Asset and } \\
\text { Vulnerability Evaluation) que tiene por objeto facilitar la evaluación de } \\
\text { riesgos en una organización [24]. }\end{array}$ \\
\hline
\end{tabular}


Continuación Tabla I. Antecedentes.

\begin{tabular}{|c|c|c|}
\hline $\begin{array}{l}\text { Juan Carlos } \\
\text { Quintero Vallejo } \\
\text { Mejía Estrada, } \\
\text { Jaime Alonso }\end{array}$ & $\begin{array}{l}\text { Estandarización de } \\
\text { procesos operacionales } \\
\text { de seguridad, } \\
\text { migración y soporte, } \\
\text { de la gerencia de TI } \\
\text { en la empresa CI } \\
\text { Banacol S. A }\end{array}$ & $\begin{array}{l}\text { BANACOL S.A. realiza varios procesos, cada uno con un fin diferente } \\
\text { dentro de su área de sistemas, entre ellos está el de administración de } \\
\text { backups, la cual es una forma de contingencia, que se realizan de forma } \\
\text { diaria de las plataformas que usa la empresa; La compañía posee una } \\
\text { gran cantidad de aplicaciones de las cuales la gran parte es producción } \\
\text { propia por tal motivo el software es diseñado acoplándose a las } \\
\text { necesidades de la empresa, esto permite que los errores detectados en las } \\
\text { diversas aplicaciones sean corregidos con mayor rapidez [25]. }\end{array}$ \\
\hline $\begin{array}{c}\text { Luis Carlos } \\
\text { Almeida } \\
\text { Montero }\end{array}$ & $\begin{array}{l}\text { Diseño y } \\
\text { documentación de un } \\
\text { plan de gestión de } \\
\text { talento humano en la } \\
\text { E.S.E hospital integrado } \\
\text { San Pedro Claver de } \\
\text { Mogotes }\end{array}$ & $\begin{array}{l}\text { En el desarrollo de este proyecto de grado se utilizaron herramientas } \\
\text { para el desarrollo de diagnósticos, la documentación de procesos y el } \\
\text { establecimiento de una estructura salarial mediante el uso de } \\
\text { herramientas estadísticas, con el objetivo de Diseñar y documentar un } \\
\text { plan de Gestión de Talento Humano en la E.S.E Hospital Integrado San } \\
\text { Pedro Claver de Mogotes orientado al mejoramiento y estandarización } \\
\text { de los procesos administrativos de la organización [26]. }\end{array}$ \\
\hline $\begin{array}{c}\text { Davis Vivente } \\
\text { Villacís } \\
\text { Navarrete }\end{array}$ & $\begin{array}{c}\text { Diseño de un sistema } \\
\text { de gestión por procesos } \\
\text { para el área de talento } \\
\text { humano de } \\
\text { Northospital }\end{array}$ & $\begin{array}{l}\text { En este proyecto el autor realiza el proceso de documentación de } \\
\text { procesos del área de talento humano en el Northospital, enfocado en el } \\
\text { modelo de competencias, con la finalidad de lograr reducir costos y } \\
\text { tiempos implicados a procedimientos dentro de la misma, para dicho } \\
\text { proceso desarrollo tres actividades en cada área para establecer puntos } \\
\text { fuertes y debilidades, con la finalidad de Realizar un plan de } \\
\text { implementación de procedimientos en Northospital, para que de esta } \\
\text { forma puedan trabajar bajo un sistema de procesos con el fin de que la } \\
\text { compañía reduzca los tiempos y costos que tienen al momento de } \\
\text { realizar las actividades del Área de Talento Humano [27]. }\end{array}$ \\
\hline $\begin{array}{l}\text { Neffer Arizala } \\
\text { Valencia, Ana } \\
\text { Maria Gómez } \\
\text { Penagos, } \\
\text { Jennifer Carolina } \\
\text { Mora Pino, } \\
\text { Mildred Gisela } \\
\text { Mora Pino, Betty } \\
\text { Alexandra } \\
\text { Vigoya Martínez }\end{array}$ & $\begin{array}{l}\text { Caracterización de la } \\
\text { gestión del talento } \\
\text { humano en la ESE } \\
\text { Norte II puntos de } \\
\text { atención Caloto y } \\
\text { Corinto Cauca }\end{array}$ & $\begin{array}{l}\text { La evaluación del área encargada de la gestión del talento humano es } \\
\text { importante para cualquier organización, partiendo de esto los autores de } \\
\text { este artículo desarrollaron este proceso de diagnóstico mediante el uso } \\
\text { de entrevistas, encuestas y listas de chequeo, desarrollándolas a las } \\
\text { personas de área de coordinación del talento humano, todo esto con el } \\
\text { fin del conocimiento del estado actual del mismo, y de esta manera } \\
\text { generaron un informe final donde se evidenciaron puntos fuertes y } \\
\text { aspectos por mejorar, para la mejora del área de talento humano y } \\
\text { correspondiente mejoramiento de la empresa, ya que esta área hace parte } \\
\text { de la base de la empresa junto con un factor financiero y la estructura } \\
\text { física, para lograr caracterizar el proceso de gestión de talento humano } \\
\text { en la ESE Norte punto de atención Caloto Cauca durante el primer } \\
\text { semestre del año } 2017 \text { [28]. }\end{array}$ \\
\hline $\begin{array}{c}\text { Gloria Arbeláez, } \\
\text { Nelson } \\
\text { Gavilánez, } \\
\text { Diana Verdezoto }\end{array}$ & $\begin{array}{c}\text { Documentación de } \\
\text { procesos de la unidad } \\
\text { de talento humano, } \\
\text { hospital Enrique Garcés }\end{array}$ & $\begin{array}{l}\text { En este documento los autores exponen la manera en la que } \\
\text { desarrollaron el proceso de estandarización en el área de talento humano } \\
\text { vinculada al hospital Enrique Garcés para Evaluar la gestión y } \\
\text { documentar los procesos estandarizados de la Unidad de Talento } \\
\text { Humano para contribuir a que su personal desarrolle las actividades } \\
\text { asignadas [29]. }\end{array}$ \\
\hline
\end{tabular}

Fuente: Los autores, 2020.

Para esta ocasión, la intención fue conocer el estado actual de la gestión documental, se debe tener en cuenta que, al inicio del proyecto, lasáreas de Gestión Humana y Seguridad y Salud en el Trabajo tenían una gestión independiente; el estado original de los documentos se visualiza en la tabla II.
Gracias a la información anterior, se elaboró el análisis FODA, matriz utilizada para identificar los riesgos y oportunidades en la organización [32], el principal objetivo es ofrecer un claro diagnóstico para poder tomar las decisiones estratégicas oportunas y mejorar en el futuro. Su nombre deriva del acrónimo formado por las iniciales

Rev. Ingeniería, M atemáticas y Ciencias de la Información Vol. 7 / N úm. 14 / julio - diciembre de 2020; pág. 77-93 


\section{OBJETIVOS}

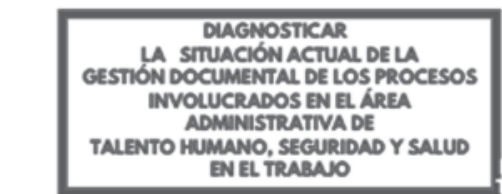

\section{ACTIVIDADES}
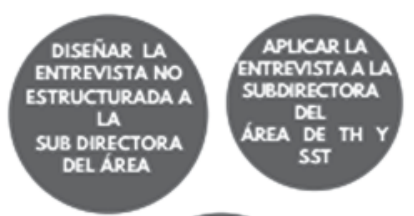

VALOARLA

ENTREVISTA

REAUZADA ALA

SUB DIRECTORA
DEL AREA DE THY

SST

\section{MECANISMO DE RECOLECCIÓN DE DATOS}
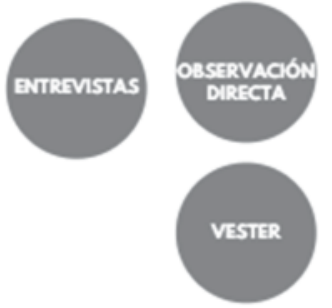
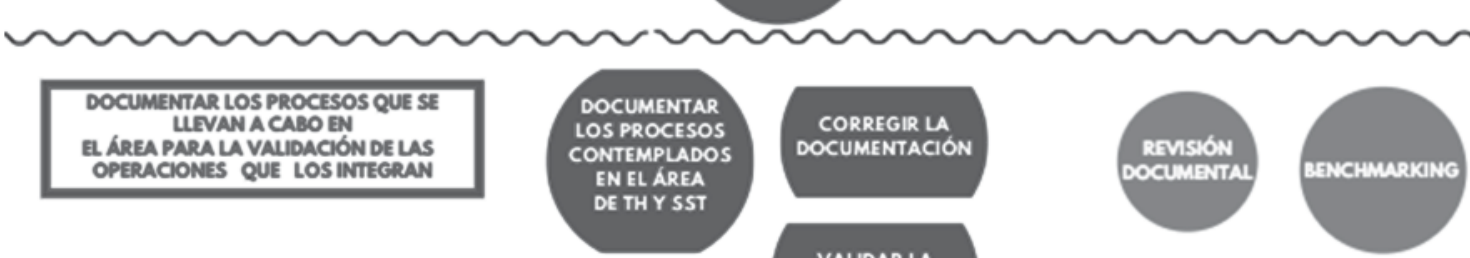

VALIDAR LA DOCUMENTACIÓN
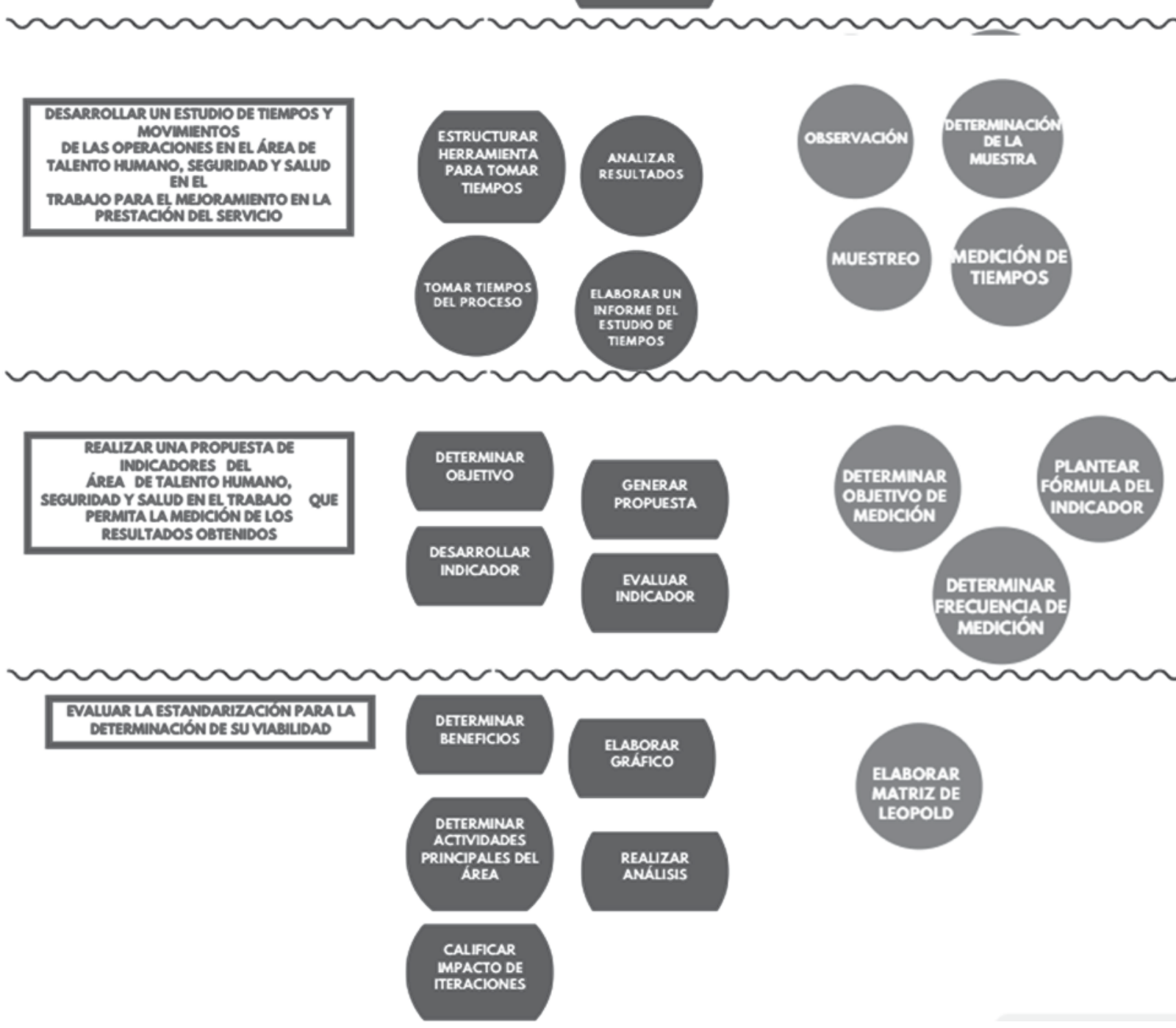

Fig. 1. Metodología. Fuente: Los autores, 2020. 
Tabla II. Gestión documental original del área de Gestión Humana y Seguridad y Salud en el trabajo.

\begin{tabular}{|c|c|c|}
\hline \multirow{2}{*}{$\begin{array}{c}\text { Tipo de } \\
\text { documento }\end{array}$} & \multicolumn{2}{|r|}{ Área } \\
\hline & $\begin{array}{l}\text { Gestión } \\
\text { Humana }\end{array}$ & $\begin{array}{l}\text { Seguridad y Salud } \\
\text { en el Trabajo }\end{array}$ \\
\hline Manuales & 3 & 0 \\
\hline Procedimientos & 8 & 15 \\
\hline Formatos & 17 & 11 \\
\hline Anexos & 15 & 0 \\
\hline $\begin{array}{l}\text { Manuales de } \\
\text { cargo }\end{array}$ & 1 & 0 \\
\hline Total & 44 & 26 \\
\hline
\end{tabular}

Fuente: Los autores, 2020

de los términos: debilidades, amenazas, fortalezas y oportunidades [33].

Para tener una visión más objetiva, seaplica una matriz Vester, la cual facilita la identificación y la determinación delas causas y consecuencias deuna situación o problema [34], inicialmente se describen los problemas principales y se les designa un código alfanumérico del 1 al 10 acompañado dela letra P, como se muestra en la tabla III.

Tabla III. Problemas iniciales para la matriz de Vester.

\begin{tabular}{|c|c|}
\hline No. & Problema \\
\hline P1 & Duplicidad de actividades \\
\hline P2 & Incumplimiento de requisitos legales \\
\hline P3 & Documentación sin actualizar \\
\hline P4 & Desconfianza en el proceso \\
\hline P5 & $\begin{array}{c}\text { Separación entre los procesos de la } \\
\text { organización }\end{array}$ \\
\hline P6 & Alta rotación de personal \\
\hline P7 & Resistencia al cambio \\
\hline P8 & No existe un seguimiento de los objetivos \\
\hline P9 & Algunos procesos sin documentar \\
\hline P10 & Insatisfacción de los colaboradores \\
\hline
\end{tabular}

Fuente: Los autores, 2020.

Básicamenteseenfrentan los problemas (Variables) entre sí basándose en los siguientes criterios decalificación:

- 0: No lo causa

- 1: Lo causa indirectamente o tiene una relación de causalidad muy débil
- 2: Lo causa de forma semidirecta o tieneuna relación de causalidad media

- 3: Lo causa directamente o tiene una relación de causalidad fuerte

Luego deesto, sesuman las filas y las columnas y se realiza un esquema axial para determinar la influencia que tienen los problemas sobre los factores descritos, como se aprecia a continuación. Figura 2.

Los problemas críticos tienen un total de activos y pasivos altos [35], es decir que los puntos que más requieren atención son: Ia duplicidad de actividades, la documentación sin actualizar, la desconfianza en el proceso y la insatisfacción de los colaboradores por lo tanto requieren un tratamiento especial, están en un punto de equilibrio entre las causas y consecuencias; los criterios activos, influyen sobre los demás, pero no son causados por otros, en este cuadrante solo se visualiza el incumplimiento de requisitos legales. Los criterios pasivos no influyen de manera importante sobre los otros, pero son causados por los demás y se denominan efectos, deacuerdo a la figura 2 se observa la inexistencia de seguimiento de los objetivos, por último, se encuentran los criterios indiferentes que no tienen ningún efecto de causalidad ni de consecuencia [36].

Con base a lo anterior, se resaltó la necesidad de comenzar a implementar medidas y planes de mejora, todo resumido a la estandarización.

\section{B. Documentación de procesos y ajuste de indicadores}

Luego de recopilar toda la información se elabora un listad o maestro de documentos donde se encuentra el nombre del subproceso al que pertenece el documento, el tipo de documento (Procedimiento, formato, plan, cronograma, programa, instructivo, anexo o manual), nombre, código según las políticas dela organización, versión, responsable del uso, nombre de las personas encaradas para revisar y aprobar lainformación; la función principal de esta actividad fue tener control y claridad sobre la información original y la información queseactualizó; como paso siguienteseinició el desarrollo dela caracterización, lo cual semanifiesta como una hoja devida queindica el objetivo 


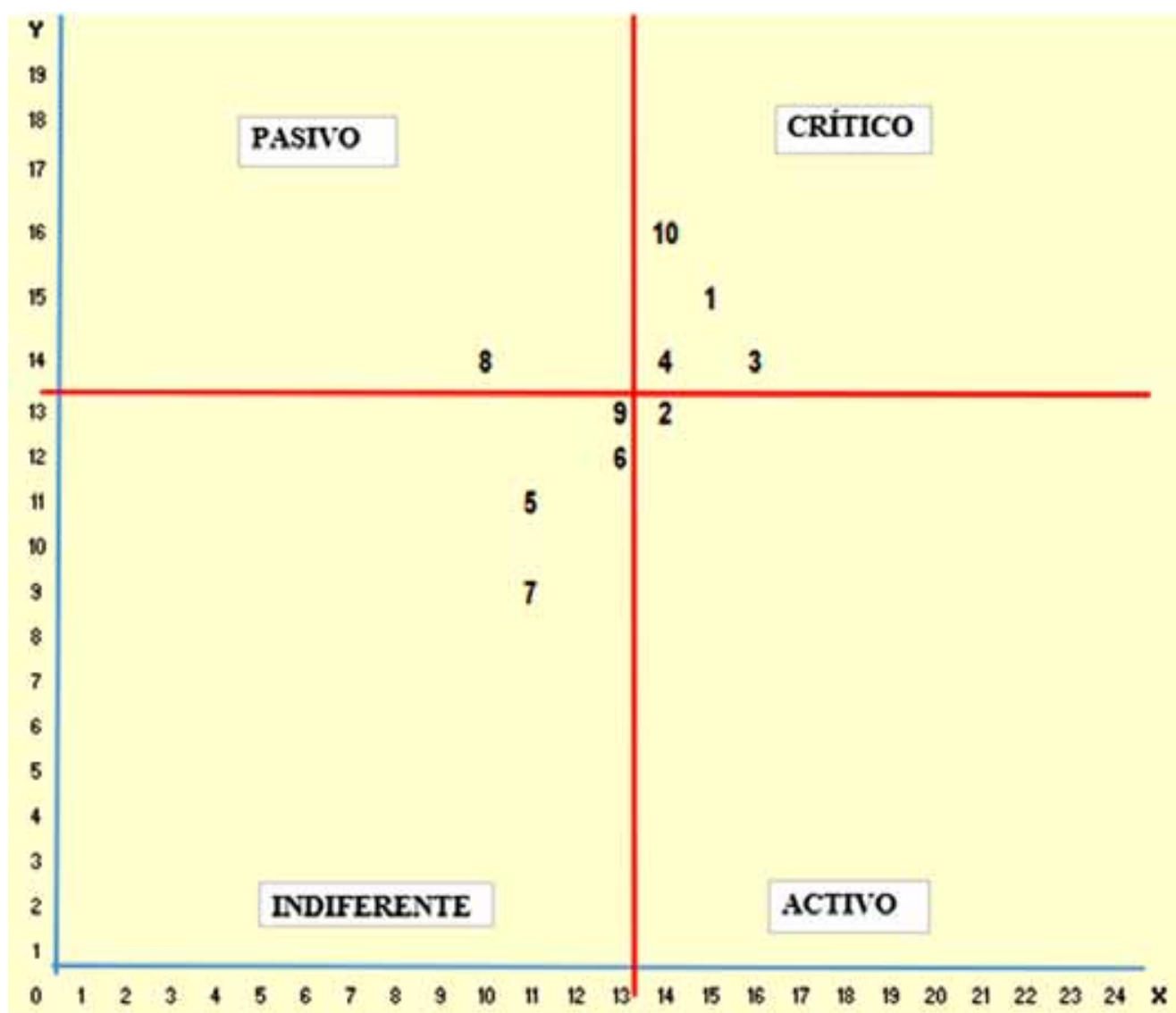

Fig. 2. Matriz Vester. Fuente: Los autores, 2020.

del área, el alcance y clasificar entradas y salidas de proveedores y clientes, separándolas en las actividades del ciclo PHVA, estas letras forman un acrónimo compuesto por las iniciales de las palabras Planificar, Hacer Verificar y Actuar [37].

Se procede a realizar el mapa de riesgos, donde se incluyen los procesos, subprocesos, procedimientos, características del riesgo y tipo decontrol ya sea preventivo, correctivo o detectivo; con la probabilidad e impacto se establece la severidad del riesgo inherente o residual mediantela cual se logra cuantificar los niveles de cada uno [38] y se permite establecer un plan de mejora que mitigue el factor que pone en riesgo la ejecución de las actividades de la organización; para el análisis dela severidad del riesgo, el representante del área tuvo en cuenta la tabla IV.

Para observar de una forma más organizada los resultados obtenidos por las calificaciones brinda- das por la subdirectora del área de Gestión Humana y SST, se realizó una gráfica clasificando los riesgos intrínsecos de cada actividad, sin tener en cuenta los controles que de éste se hagan a su interior o riesgos residuales [39] como se observa en la Fig. 3.

La elaboración de la matriz de riesgo sirvió de base para la estructuración de la documentación del área, sin embargo, cabe resaltar que, durante este proceso, al gunos documentos estaban sujetos a modificaciones debido a cambios organizacionales.

Toda la información documentada cuenta con una estructura definida por la institución, por ejemplo, todos los procedimientos, manuales einstructivos deben llevar un objetivo, alcance, terminología y definiciones (Si aplica), un diagrama de flujo que ayudará a visualizar y mejorar estos procesos y en definitiva a llevar una mejor ges- 
Tabla IV. Severidad del riesgo.

\begin{tabular}{|c|c|}
\hline $\begin{array}{l}\text { Nivel de } \\
\text { exposición }\end{array}$ & Descripción \\
\hline Extremo & $\begin{array}{l}\text { INTERVENCIÓN URGENTE: } \\
\text { Realizar plan de mejoramiento } \\
\text { inmediato. Intervención del riesgo } \\
\text { en el término de máximo de un (1) mes } \\
\text { después de realizada la auditoría }\end{array}$ \\
\hline Alto & $\begin{array}{l}\text { CORREGIR Y ADOPTAR } \\
\text { RECOMENDACIONES DE } \\
\text { INMEDIATO: } \\
\text { Realizar plan de mejoramiento } \\
\text { inmediato. Intervención del riesgo } \\
\text { en el término de máximo de tres (3) mes } \\
\text { después de realizada la auditoría }\end{array}$ \\
\hline Moderado & $\begin{array}{l}\text { ADOPTAR PLAN DE } \\
\text { MEJORAMIENTO - MEJORAR } \\
\text { SI ES POSIBLE: } \\
\text { Realizar plan de mejoramiento } \\
\text { inmediato. Intervención del riesgo en el } \\
\text { término de máximo de tres a seis ( } 3 \text { - 6) } \\
\text { mes después de realizada la auditoría. }\end{array}$ \\
\hline Bajo & $\begin{array}{l}\text { MANTENER O CONSIDERAR } \\
\text { SOLUCIONES - REALIZAR } \\
\text { COMPROBACIÓN PERÍODICA } \\
\text { PARA ASEGURAR } \\
\text { CUMPLIMIENTO: } \\
\text { Mantener la calificación e incluir en el } \\
\text { Plan Anual de Trabajo las mejoras } \\
\text { detectadas. }\end{array}$ \\
\hline
\end{tabular}

Fuente: Los autores con base en la ISO 31000, 2020 tión empresarial [40] (Si aplica) y un cuadro de control de cambios donde se relacionen las versiones con la descripción de las modificaciones realizadas con el fin de es real izar el seguimiento integrado modificaciones llevadas a cabo para su supervisión [41]. La definición de los objetivos del área permite el ajuste y complemento de los indicadores, puesto que, debe haber claridad sobre las prioridades (Objetivos estratégicos) de la empresa, para queel ejercicio conllevea procesos de mejora [42].

Los indicadores tienen como requisito responder las preguntas de ¿qué?, ¿cómo?, ¿cuándo?, ¿dónde? y ¿por qué? [43] . Adicionalmente debe contener en su estructura: nombre, objetivo, fórmula, fuentes de información, periodicidad de medición y de reporte, todos estos datos se visualizan en la hoja devida de cada indicador, en el formato estructurado por la organización.

\section{Estudio de tiempos y movimientos}

El estudio del trabajo tiene por objeto examinar de qué manera se está realizando una actividad, simplificar o modificar el método operativo para reducir el trabajo innecesario o excesivo y fijar el tiempo normal parala realización deesa actividad [44]. Inicialmente, Taylor y los esposos Gilbreth fueron los principales precursores deesta metodología basa en los tiempos estándar de un colaborador para proporcionar confiabilidad y re sultados de alta calidad [45].

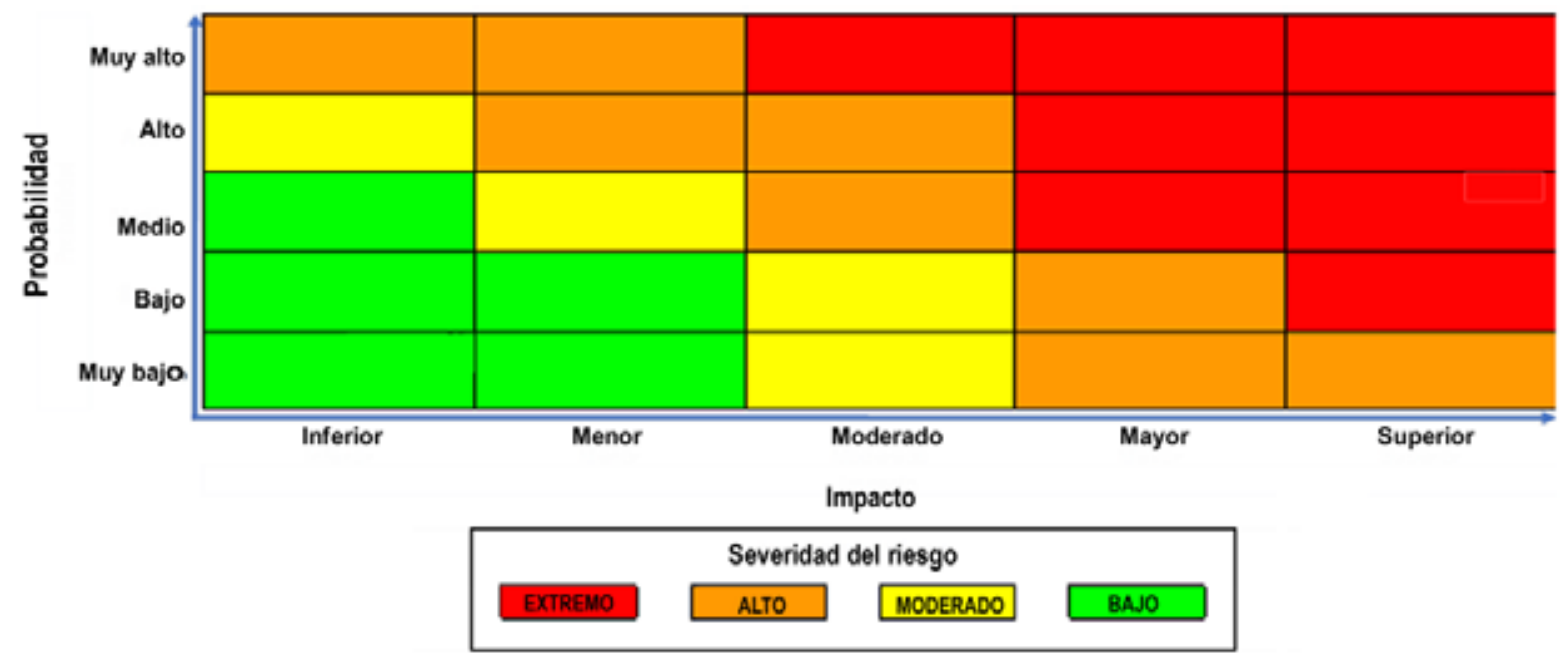

Fig. 3. Matriz de riesgo. Fuente: LoS autores con base en la ISO 31000, 2020. 
El estudio realizado en el área de Gestión Humana y Seguridad y Salud en el Trabajo, se inicia con el análisis previo de las actividades de cada uno de los procedimientos, a través de la consolidación en diagramas de operaciones como el presentado en la Fig. 4.
Luego deesto, seestableció la herramienta para la medición correspondiente, la cual fue la del cálculo de los tiempos estándar mediante el formato de la figura 5 , en la cual se consolida el tiempo establecido por el encargado del área en la organización analizaday dos "expertos" adicionalesque

\section{Inducción, reducción y entrenamiento del talento humano}

Necesidad de inducción,
reducción y entrenaimiento

Realizar inducción general $^{N R}$

Instrucciones y entrega de formato de entrenamiento del puesto de trabajo

Entrenamiento del puesto de trabajo

Reentrenamiento

entrega

NR

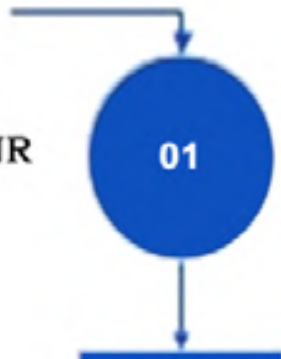

Tiempo promedio

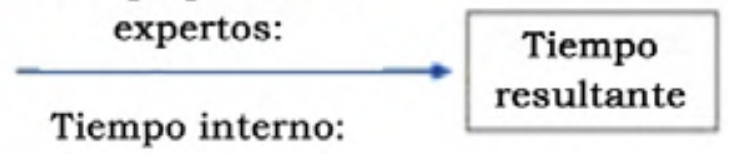

\section{Tiempo promedio}

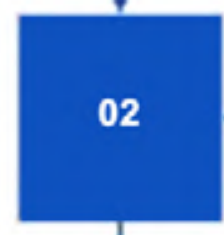

expertos:

Tiempo interno:

Tiempo resultante

Tiempo promedio

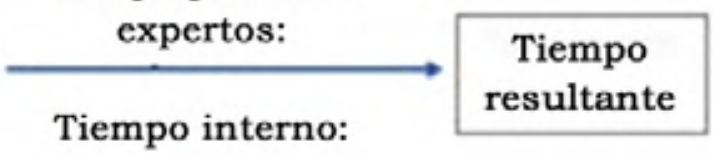

Tiempo promedio

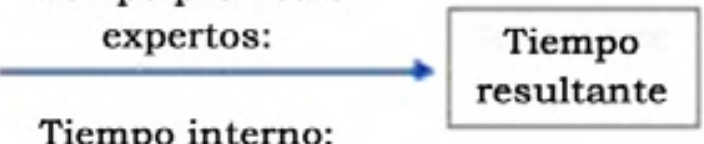

Tiempo interno:

Tiempo promedio

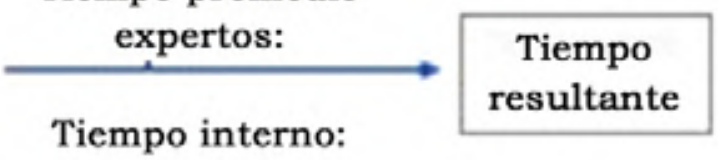

Tiempo promedio

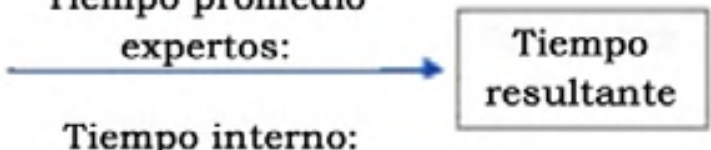

Tiempo interno:

Fig. 4. Diagrama de operaciones. Fuente: Los autores, 2020. 


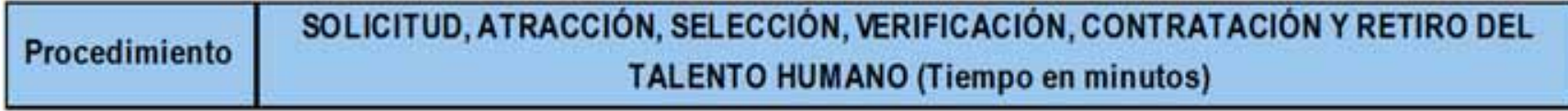

\begin{tabular}{|c|c|c|c|c|c|}
\hline Paso & Nombre del paso & 0 & $\mathbf{1}$ & $\mathbf{2}$ & $\begin{array}{c}\text { Tiempo } \\
\text { Estándar (Min) }\end{array}$ \\
\hline 1 & Identificar las necesidades de talento humano & 5 & 5 & 5 & 5 \\
\hline 2 & Aprobar solicitud de vacante & 480 & 240 & 480 & 408 \\
\hline 3 & Definir tipo de contrato y correspondiente salario & 5 & 7 & 6 & 5.9 \\
\hline 4 & Publicación de la vacante & 15 & 20 & 10 & 15 \\
\hline
\end{tabular}

Fig. 5. Tiempos estructurados. Fuente: Los autores, 2020.

a través de su conocimiento y experiencia brindaron un valor aproximado en minutos para cada una de las actividades.

En cada medición se le estableció un porcentajea cada valor, directamente proporcional a cada uno de Iosconocimientosy experienciaqueel experto en cuestión tiene, generando así la siguienteecuación (1):

TE: $(\mathrm{TL} * 40 \%)+(\mathrm{T} 1 * 30 \%)+(\mathrm{T} 2 * 30 \%)$

Siendo

TE: Tiempo estándar

TL: Tiempo encargado del área analizada

T1: Tiempo experto 1

T2: Tiempo experto 1

\section{Estudio de impacto}

Finalmente, se observa la necesidad de evaluar el impacto y la viabilidad quetuvo el proyecto frente a las necesidades planteadas inicialmente. Se aplica la matriz de Leopold, un cuadro de doble entrada de relación causa-efecto empleado en la evaluación del alcance, esta matriz sistematiza la relación entrelas acciones a implementar en la eje cución de un proyecto y su posible efecto [46].

Para la elaboración dela matriz, se identificaron las iteraciones comparables y calificables existentes, seconsideraron las actividades principales del área, tales como los subprocesos (Columnas) que serán impactadas por los factores asociados que setoman como los beneficios esperados al desarrollar el proyecto (Filas); en cuanto a la valoración del riesgo, se determina que, si se presentan resultados nega- tivos, se consideran perjudiciales para la organización, de acuerdo a la calificación de los ítems brindada por la subdirectora del área de Gestión humana y SST; se debe tener en cuenta que para la magnitud y gravedad del impacto se asignaron valores deforma subjetiva por el encargado del área.

Se debe resaltar que gracias al manejo y orden con el queel profesional encargado ha ejecutado las actividades requeridas la organización maneja un excelente clima laborar logrando así un buen nivel de satisfacción a su cliente interno y externo; la estandarización aquí planteada complementa deuna forma positiva la empresa, debido a que a medida que surjan procedimientos nuevos la subdirectora del áreaya manejará el estándar y la estructura brindada por el proyecto, lo cual lepermite mayor control sobre sus tareas; en cuanto al ajuste de indicadores, es posible mantener trazabilidad y hacer seguimiento de los objetivos planteados; el formatos de éstos fue suministrado directamente por la organización.

La evaluación deimpacto permitecuantificar con rigurosidad científica el efecto atribuible a una intervención específica. A plicadaala política pública, ofrece la oportunidad de conocer el alcance de las iniciativas [47], la viabilidad de un proyecto queda determinada en un caso de negocio que se expresa en términos de un conjunto de beneficios que contribuyen hacia el objetivo estratégico [48], para visualizarla con baseen la puntuación brindada por el delegado del área en la matriz de Leopold, no se observa ningún puntaje con val or menor a cero, los hallazgos positivos se traducen en oportunidades de mejora, los beneficios que serán de mayor utilidad serán los que se muestran en la tabla V. 
Tabla V. Porcentajes de utilidad según beneficio

\begin{tabular}{|l|l|c|}
\hline$\#$ & \multicolumn{1}{|c|}{ Beneficio } & Porcentaje de utilidad (\%) \\
\hline 2. & Evitar duplicidad de actividades & 68,75 \\
\hline 5. & Reducción de errores & 66,67 \\
\hline 9. & Aporte a la adherencia de los colaboradores & 66,67 \\
\hline 1. & Mejorar cumplimiento en la ejecución de los procedimientos & 62,5 \\
\hline 4. & Cumplimiento de requisitos legales & 60 \\
\hline 6. & Documentación actualizada & 60 \\
\hline 8. & Mayor confiabilidad del proceso & 60 \\
\hline 10. & Mejorar desempeño de los colaboradores & 60 \\
\hline 7. & Integralidad entre los procedimientos de la organización & 50 \\
\hline 3. & Facilitar revisión de auditoría & 50 \\
\hline
\end{tabular}

Fuente: Los autores, 2020.

El número de iteraciones positivas se deriva de la cantidad de valores mayores a cero de la calificación de magnitud e importancia por subproceso del área, como se visualiza en la matriz de Leopold para este proyecto no se generan valores negativos, por lo que se cuentan 2 iteraciones positivas por beneficio de la estandarización. El resultado del promedio del proyecto es de 23.67/ 30, si se obtiene el porcentaje de este valor, se adquiere el $78.9 \%$ de impacto del propósito del trabajo de grado.

\section{Discusión De RESUltados}

Con base en la búsqueda de casos de estudio que manejan una normalización de actividades en organizaciones similares, se toma como referencia inicial la estandarización de procesos críticos de la central de esterilización del hospital san José para subprocesos delimpieza y descontaminación según Ios requisitos de la NTC ISO 9001-2015, proyecto realizado por una estudiante de la Universidad Católica de Colombia; la metodología empleada consistió en recolectar información acerca de los procesos que se desarrollaban en la entidad, por medio deentrevistas eindagación en página oficial; para el diseño del plan de actividades, se desarroIló la caracterización de la central de esterilización, luego sedocumentaron los procesos apoyándoseen la norma y su formato decumplimiento; concluyendo, seajustaron losindicadores degestión para realizar seguimiento a los procesos [49]; como segunda referencia se validó la estandarización y documen- tación de procesos del centro de investigación en anomalías congénitas y enfermedades raras (CIACER), estudio ejecutado por dos estudiantes de la universidad ICESI, la estrategia de trabajo consistió inicialmente en diagnosticar el área, llevando a cabo reuniones con el encargado del proceso y creando un análisis DOFA para las actividades principales que se real izaban, allí secondensó la información encontrada en las entrevistas y encuestas realizadas, luego de esto se realizó una matriz de priorización y posteriormente se elaboró la caracterización de las tareas del área, como paso siguiente se documentó la información en los formatos establecidos por la organización induyendo diagramas deflujo, al cance, propósito, términos claves y paso a paso de las actividades; finalmente, para validar el impacto del proyecto, real izaron una evaluación a los principales involucrados, quienes brindaron calificaciones acerca de si la estandarización fue o no acertada [50].

Con relación al presenteartículo, sevisualiza una estrategia similar de ejecución, teniendo en cuenta el tema principal queconsistía en la estandarización de procesos en entidades de salud, en cuanto a la fase inicial se visualiza el manejo de entrevistas a los encargados de los procesos y la construcción de una caracterización, finalizando, seadoptó la estrategia de la primera referencia en cuanto a como se aprecia en los proyectos, de esta manera, sedemostró la concordancia de actividades, adicionando el estudio de tiempos y movimientos y la viabilidad del proyecto mediantela matriz de Leopold. Finalmente, el resultado de el caso de estudio se puede 
comparar con los beneficios demostrados en las empresas anteriormente mencionadas, cabe resaltar quetodo se realizó en pro dela optimización de las áreas afectadas.

\section{CONCLUSIONES Y RECOMENDACIONES}

Con base en el trabajo realizado en el área de Gestión Humana y SST, además deidentificar riesgos y posibles amenazas con la fase inicial del caso de estudio, adicional a esto, se complementó la herramienta para reconocer las incidencias más fácilmente y poderles hacer su respectivo control y seguimiento, con la anterior información sehace referencia a los indicadores.

Al día de hoy, el área cuenta con uniformidad en sus formatos, procedimientos, manuales y anexos, debido a que, todos los documentos mencionados son homogéneos en cuanto a su estructura visual, lo que permite una mejor comprensión tanto para el encargado del proceso como de cualquier clienteinterno o externo que desee comprender los procedimientos o que requiera acceder a esta información; en este sentido, se entregó un listado maestro de documentos, el cual contiene los títulos y codificaciones dela gestión documental del área, serecomienda a la entidad oncológica que este archivo sea actual izado a medida que se creen más documentos o queel área se modifique, con el propósito de no perder trazabilidad y mantener el orden en las actividades.

Final mente, secomplementaron y ajustaron los indicadores de gestión en su respectiva hoja de vida (Estructura brindada por la entidad oncológica), que proporcionarán información verídica para el gestor cuando necesite saber el estado de las actividades de la organización, conviene enfatizar que este ajuste permitirá una mayor precisión en la toma acertada de decisiones y mayor transparencia a la hora de comunicar resultados cuando se requiera, se aconseja, mantener este elementeal día con la información semestral, mensual o anual según lo señale el indicador.

\section{Referencias}

[1] J. A. Martínez, «Alborum»https:/ / www.alborum. com/ la-estandarizacion-los-procesos-garantiaexito-industrial/ . 2016.
[2] Ingrandey Tiziana, «Estandarizar: trabajar deforma organizada y controlada» Kailean consultores, http:/ / kailean.es/ estandarizar-trabajar-de-formaorganizada-y-controlada/ . 2017.

[3] J. Pacheco, «Estandarización de procesos: todo lo que se necesita saber» H eflo https:/ / www.heflo. $\mathrm{com} / \mathrm{es} / \mathrm{blog} / \mathrm{bpm} /$ estandarizacion-procesos/ \#: :text=L a\%20estandarizaci \%C3\%B3n\%20 de\%20procesos\%2C\%20seg\%C3\%BA n,Definir $\% 20$ el \%20est\%C 3\%A 1nd ar\&text=Establecer \%20la\% 20adhesi\%C3\%B3n\%20al\%20Est\% C3\% A Indar,una\%20mejora\%20continua\%20del\%20 Standard. 2017.

[4] M. Riquelme, « ¿Como hacer un levantamiento de procesos en una empresa?» Web y empresas https:/ / www.webyempresas.com/ como-hacer-un-levanta miento-de-procesos-en-una-empresa/ \#Guia para_levantar_un_proceso_en_una_empresa. $201 \overline{2}$.

[5] Farias y J. María, «Gestión del capital humano» Course Hero, https:/ / www.coursehero.com/ file/ p75grug/ trabajo-Es-el-aumento-en-la-capacidad-dela-producci\%C3\%B3n-al canzadas-con-mejoras/ .

[6] S. Baez, «El capital humano y competencias» https:/ / www.eoi.es/ blogs/ madeon/ 2013/ 05/ 22/ el-capital-humano-y-competencias/ . 2013.

[7] Estrategias deinversión, «Estrategias deinversión» https:/ / www.estrategiasdeinversion.com/ actualidad/ noticias/ bolsa-espana/ que-son-losindicadores-y-para-que-sirven-n-229166\#. :text= Los\%20indicadores\%20son\%20variables\% 20 que,para\%20as\%C3\%A D\%2C\%20poder\%20 respaldar\%20acciones. 2012.

[8] Korporate Technologies Group, «Korporate Technologies Group»[En línea] https:/ / grupokorporate.com/ las-cinco-fases-que-debesdetener-claras-antes-iniciar-un-proyecto-de-mejora-de-procesos/ .

[9] ICONTEC, «ISOTools» [En línea]. https:/ / www. isotools.org/ 2014/ 12/ 15/ iso-9001-2015-gestionriesgo/ . 2014.

[10] N.P, «Emprende pyme.net» [En línea]. https:/ / www.emprendepyme.net/ mapa-de-riesgos-deuna-empresa.html . 2017.

[11] C. L. A., «SciElo» Instituto N acional deaprendizaje de Costa Rica. https:/ / www.scielo.sa.cr/ scielo.php?script=sci_arttext\&pid=\$2215-2458201 7000200088. 2017.

[12] IMF Business School, «El Blog de IMF Business School» https:/ / blogs.imf-formacion.com/ blog/ mba/ mapas-de-procesos-empresas-servicios/ . 2019.

[13] Instituto Europeo dePosgrado, «I nstituto Europeo dePosgrado» [En línea]. https:/ / www.iep.edu.es/ gestion-del-talento-humano-que-es/ . 2018. 
[14] N 9000. https:/ / www.normas9000.com/ content/ Glosario.aspx\#glosarioG.

[15] Ministerio de Trabajo, «MINTRA BAJO» [En línea]. https:/ / www.mintrabajo.gov.co/ relaciones-laborales/ riesgos-laborales/ sistema-de-gestion-de-seguridad-y-salud-en-el-trabajo. 2019.

[16] E. m. p. managment, «Documentos planning» [En línea]. http:/ / planning.com.co/ bd/ documentos Planning/ Agosto2001.pdf. 2001.

[17] Actualicese, https:/ / actualicese.com/ definicionde-auditoria/ . 2019.

[18] Universidad autónoma del estado de Hidalgo, https:/ / www.uaeh.edu.mx/ calidad/ docs/ calidad/ cursos/ Requisitos_Legales.pdf. 2018.

[19] Debitoor, https:/ / debitoor.es/ glosario/ definicionnomina. 2018.

[20] G. A. Guachi A y D. Tania, «Universidad Técnica de Á mbato» http:/ / redi.uta.edu.ec/ handle/ 123456789/ 2361. 2012.

[21] S. D. Martínez G y C. José, «PontificiaUniversidad Javeriana» https:/ / repository.javeriana.edu.co/ handle/ 10554/ 7532. 2010.

[22] M. Alberto y F. Julián, «SciElo,» http:/ / www. scielo.org.co/ scielo.php?script=sci arttext \&pid=S0121-11292014000200007\&lang=-pt. 2014.

[23] Q. Casadiegos y M. Aura, «Repositorio Institucional Universidad Francisco de Paula Santander deOcaña» http:/ / repositorio.ufpso.edu.co:8080/ dspaceufpso/ . 2012.

[24] P. Gómez y D. Ricardo, «Revista delngeniería Universidad Autónoma de Manizales» https:/ / www.redalyc.org/ articulo.oa?id $=121015012006$. 2006.

[25] M. Quintero y J.Juan, «Biblioteca Digital Lasallista» http:/ / repository.Iasallista.edu.co/ dspace/ handle/ 10567/ 744. 2015.

[26] L. M ontero, «Diseño y documentación de un plan degestión de talento humano en la E.S.E hospital integrado San Pedro Claver de Mogotes» p. 15, 2015.

[27] D. Navarrete, «Diseño de un sistema de gestión por procesos para el área de talento humano de northospital» http:/ / repositorio.puce.edu.ec/ handle/ 22000/ 10727. 2015.

[28] M. N effer A rizala Valencia, «Caracterización dela gestión del talento humano puntos de atención caloto y corinto Cauca» http:/ / 200.21.94.179: 8080/ jspui/ bitstream/ handle/ 10839/ 1834/ $\mathrm{N}$ effer\%20A rizal a\%20Val encia.pdf?sequence $=1 \&$ isAllowed $=y .2017$.
[29] N. Gloria A rbeláez, «Documentación de procesos de la unidad de talento humano, HOSPITAL EN RIQUE GARCÉS» http:/ / repositorio.espe.edu.ed/ bitstream/ 21000/ 6030/ 1/ A C-A GH-ESPE033868.pdf. 2012

[30] «La investigación» https:/ / lainvestigacion.com/ descriptiva/

[31] «Question Pro» https:/ / www.questionpro.com/ blog/ es/ entrevista-estucturada-y-no-estructurada/ .

[32] P.Torres, «IVE Consultores» https:/ / iveconsultores.com/ matriz-dafo/

[33] R. Espinosa, FODA https:/ / robertoespinosa.es/ 2013/ 07/ 29/ la-matriz-de-analisis-dafo-foda. 2013.

[34] A. Vallésy F. Alex, «A prendizajey desarrollo,» La cajadelashabilidades. http:/ / www.myadriapolis. net/ 2015/ 03/ la-matriz-de-vester-ejemplo-practico. html. 2015.

[35] «lngenio Empresa» https:/ / ingenioempresa.com/ matriz-de-vester/ \#. -text=Caracterizar\%20y\%20 priorizar\%20los\%20problemas,problema\%20cumpliendo \%20los\%20objetivos\%20trazados. 2016.

[36] Cañedo y Carlos, «Tesis doctoral es de economía» Eumed, https:/ / www.eumed.net/ tesis-doctorales/ 2008/ cmci/ M etodologia\%20para\%20la\% 20aplicacion\% 20de\%20la\%20MATRIZ\%20DE\% 2OVESTER.htm.

[37] «ISO Tools excellence» https:/ / www.isotools.org/ 2015/ 02/ 20/ en-que-consiste-el-ciclo-phva-demejora-continua/ . 2017.

[38] BBVA, https:/ / accionistaseinversores.bbva.com/ microsites/ informes2010/ es/ Gestiondelriesgo/ SeveridadLGD.html. 2011.

[39] I. Rodiguez, «AUDITOOL» https:/ / www. auditool.org/ blog/ control-interno/ 3073-que-esel-riesgo-riesgo-inherente-y-riesgo-residual\#: : text=En\%20auditor\%C3\%ADa\%2C\% 20\%22Riesgo \%20inherente\%22,no\%20hubo\%20controles\% 20internos\%20relacionados.. 2017.

[40] iveconsultores, https:/ / iveconsultores.com/ diagrama-de-flujo/ . 2020.

[41] UV Executivemaster project managment, https:/ / uv-mdap.com/ programa-desarrollado/ gestionde-cambios-del-proyecto/ . 2017.

[42] Roncancio y Gabriel, «Indicadores de Gestión (KPI'S): Tipos y ejemplos» https:/ / gestion. pensemos.com/ indicadores-de-gestion-tipos-yejemplos. 2019.

[43] Portal Duoc, http:/ / www.duoc.cl/ biblioteca/ crai/ redaccion-de-objetivos-en-un-trabajo-deinvestigacion. 2018.

Rev. Ingeniería, M atemáticas y Ciencias de la Información Vol. 7 / Núm. 14 / julio - diciembre de 2020; pág. 77-93 
[44] A. Kanawaty «Oficina Internacional del Trabajo Ginebra,» del ntroducción al Estudio del Trabajo, Suiza, OIT, p. 522.1996.

[45] S. P. Tejada y V. A. N oris, «M etodología deestudio detiempo y movimiento; introducción al GSD,» $3 c$ Empresa, p. 49, 2017.

[46] E. Gómez y V. Violeta, «Matriz de Leopold: para qué sirve, ventajas, ejemplos,» Lifeder, https:/ / www.lifeder.com/ matriz-deleopold/ .

[47] «5 razones para realizar una evaluación de impacto» CAF Banco de Desarrollo deA mérica Latina https:/ / www.caf.com/ es/ actualidad/ noticias/ 2017/ 02/ servidor-publico-5-razonespara-real izar-una-evaluacion-de-impacto/ .2017.

[48] «Cómo determinar la viabilidad deun proyecto,» OBS Business School, https:/ / obsbusiness. school/ int/ blog-project-management/ estrategiasclave-para-minimizar-los-riesgos-de-un-proyecto/ como-determinar-la-viabilidad-de-un-proyecto. 2018.
[49] A. Redondo y L. Constanza, «Estandarización de procesos críticos dela central de esterilización del hospital san José para subprocesos de limpieza y descontaminación según los requisitos dela NTC ISO 9001-2015,» https:/ / repository.ucatolica. edu.co/ bitstream/ 10983/ 23595/ 1/ ESTANDARIZACI\%C3\%93N\%20DE\%2OPROCE SOS\%20CR\%C3\%8DTICOS\%20DE\%20LA \%20 CENTRA L \%20DE\%20ESTERILIZA CI\%C 3\%93 N\%20DEL\%20H OSPITAL\%20SAN \%20JOS\%C 3\% 89\%2OPA RA\%20S.pdf. 2019.

[50] M. Corzo y M. Karol, «Estandarización y documentación de procesos del centro de investigación en anomalías congénitas y enfermedades raras (CIACER),» 2019. https:/ / repository.icesi.edu.co/ biblioteca_digital/ bitstream/ 10906/ 84939/ 1/ TG02570.pdf. 
